\title{
SETS OF LOCAL SEPARATING POINTS OF A CONTINUUM*
}

\author{
BY G. T. WHYBURN
}

1. Introduction. Let $M$ denote any locally compact metric continuum and let $L$ be the set of all local separating points $\dagger$ of $M$. We proceed to establish the following six properties, of which, for our immediate purposes, the most useful is number (iv).

(i). If $U$ is any uncountable subset of $L$, there exists a point $x$ of $U$ which is a point of order 2 in $M$ relative to $U$.

This statement means that $x$ is contained in arbitrarily small neighborhoods whose boundaries have in common with $M$ just two points and these two points belong to $U$. A proof has already been given by the author (loc. cit.).

(ii). If $H$ is any connected subset of $M$, then $(\bar{H}-H) \cdot L$ is countable.

For if not, (i) would give a point $x$ of this set which could be separated in $M$ from some point of $H$ by two points not in $H$, which obviously is impossible since $H+x$ is connected.

(iii). If $H$ is any connected subset of $M$, the points of $H \cdot L$ which are not local separating points of $H$ are countable.

This results immediately from (i).

(iv). If $H$ is any connected subset of $M$ such that $\bar{H} \subset L+C$, where $C$ is some countable set, then $H$ is a locally connected $G_{\delta^{-}}$ set. Hence $H$ is arcwise connected.

By (ii) we see that $(\bar{H}-H) \cdot L$ and hence $\bar{H}-H$ itself is countable. Thus $H$ is a $G_{\delta}$-set. Now $\bar{H}$ must be a regular curve, for by (i), all save a countable number of its points are points of order 2. Thus any connected subset of $\bar{H}$, and in particular $H$, is locally connected. That $H$ is arcwise connected follows now by the well known theorem of Moore-Menger. $\ddagger$

* Presented to the Society, February 25, 1933.

$\dagger$ A point $p$ is a local separating point of $M$ provided some neighborhood $V$ of $p$ exists such that $M \cdot \bar{V}-p$ is separated between some pair of points belonging to the component of $M \cdot \bar{V}$ which contains $p$. See the author's paper in Monatshefte für Mathematik und Physik, vol. 36 (1929), pp. 305-314.

$\ddagger$ See R. L. Moore, Foundations of Point Set Theory, Colloquium Publications of this Society, vol. 13 (1932), p. 86; and K. Menger, Monatshefte für Mathematik und Physik, vol. 36 (1929), pp. 193-218. 
(v). If $N$ is any continuum $\subset L+C$, where $C$ is countable, then every connected subset of $N$ is a locally connected $G_{\delta}$-set and hence is arcwise connected.

This is a corollary to (iv).

(vi). If $M$ is locally connected, then every connected subset $H$ of $L$ is the difference between an $F_{\sigma}$ and a countable set.

For it is known* that in this case $L$ is an $F_{\sigma}$, so that $M-L$ is a $G_{\delta}$ and hence so also is $(M-L) \cdot \bar{H}=(M-L) \cdot(\bar{H}-H)$. Whence, $H=[\bar{H}-(M-L) \cdot(\bar{H}-H)]-L \cdot(\bar{H}-H)$, and the first of these two sets is an $F_{\sigma}$ and the second, by (ii), is countable.

2. THEOREM. In order that every connected subset of a continuum $M$ be a $G_{\delta}$ it is necessary and sufficient that the set $N$ of non-local-separating points of $M$ be countable.

The sufficiency of the condition results immediately from (iv), in view of the fact that, for any connected subset $H$ of $M$, we have $\bar{H} \subset M=L+N$, and $N$ is countable.

To prove that the condition is necessary, we suppose, on the contrary, that $N$ is uncountable. Let $M=M_{1}+M_{2}$, where $M_{1}$ and $M_{2}$ are disjoint and totally imperfect $\dagger$ and where $M_{2}$, say, contains uncountably many points of $N$. Then $\ddagger M_{1}+L$ is connected and $M-\left(M_{1}+L\right)=E$ is totally imperfect and uncountable. Thus $E$ is not an $F_{\sigma}$ and hence $M_{1}+L$ is not a $G_{\delta}$.

Corollary 1. If every connected subset of $M$ is $a G_{\delta}$, then $M$ is a regular curve, no cyclic element of $M$ has a continuum of condensation, and the end points of $M$ are countable.

COROLlary 2. If all save a countable number of the points of each cyclic element $C$ of a locally connected continuum $M$ are local separating points of $C$, then every connected subset of $M$ is arcwise connected.

Corollary 3. If the non-local-separating points of each cyclic element of a locally connected continuum $M$ are countable, then every connected subset of $M$ will be $a G_{\delta}$ if and only if the end points of $M$ are countable.

* See the author's paper, Mathematische Annalen, vol. 162 (1929), p. 318.

$\dagger$ That is, neither contains any perfect set. For a proof that such a division of $M$ is possible, see F. Bernstein, Leipziger Berichte, vol. 60 (1908), p. 325 and Hausdorff, Mengenlehre, 1927, p. 156.

$\ddagger$ See my paper On the existence of totally imperfect sets . . . , American Journal of Mathematics, vol. 55 (1933), pp. 146-152. 
For any local separating point of a cyclic element of $M$ is a local separating point of $M$ and any non-local-separating point of $M$ which is on no non-degenerate cyclic element of $M$ must be an end point of $M$.

3. THEOREM. If $M$ is any locally compact continuum such that (a) no two maximal free arcs* in $M$ abut and (b) $L \cdot \bar{R}$ is countable and $R \neq 0$, where $R$ is the set of all ramification points (that is, points of order $>2$ ) of $M$, then $M$ contains a connected subset which is not arcwise connected.

Proof. Let $M=M_{1}+M_{2}$, where $M_{1}$ and $M_{2}$ are totally imperfect and disjoint. Set $E=M_{1}+L$. Then since $M-E \subset M_{2}, E$ is connected (loc. cit.). Furthermore $E \cdot \bar{R}=M_{1} \cdot \bar{R}+L \cdot \bar{R}$, and since $L \cdot \bar{R}$ is countable it follows that $E \cdot \bar{R}$ is totally imperfect. Now if $a$ and $b$ are two points of $E$ lying in different maximal free $\operatorname{arcs} \dagger$ of $M$, there can exist no arc $a b$ in $E$. For if $a b$ is any arc in $M$ from $a$ to $n$, then $a b \cdot \bar{R}$ cannot be countable, (for if so, some two free arcs contained in $a b$ would abut), and hence it must contain a perfect set. Thus $a b$ cannot be $\subset E$, since $E \cdot \bar{R}$ is totally imperfect.

4. ExAmple. There exists a regular curve $C$ such that (a) no two free arcs of $C$ abut, (b) $L \cdot \bar{R}$ is countable, and (c) $\bar{R}$ is punctiform. Hence $C$ contains a connected subset which is not arcwise connected.

Let $I$ be the unit interval and let $K$ be the non-dense perfect set consisting of all numbers on $I$ which can be expressed in the triadic number system using only the digits 0 and 2 . Let $I_{1}$, $I_{2}, \cdots$, be the segments on $I$ complementary to $K$ ordered in descending order of length and let $p_{1}, p_{2}, \cdots$, be the end points of these segments, where $p_{2 n-1}$ and $p_{2 n}$ are the end points of $I_{n}$. Let $a_{n}=\sum_{1}^{n} 2^{m}$ and $P_{n}=\sum_{1}^{a_{n}} p_{i}$ and $P=\sum_{1}^{\infty} P_{i}$. Let us select in $K-P$ two sequences of points $x_{n}$ and $y_{n}$ such that

$$
\begin{aligned}
0 \leftarrow \cdots<x_{n}<x_{n-1}<\cdots & <x_{1} \\
& <y_{1}<\cdots<y_{n-1}<y_{n}<\cdots \rightarrow 1 .
\end{aligned}
$$

* An arc $a b$ is free in $M$ provided $a b-(a+b)$ is an open subset of $M$. Two such arcs are said to abut if they have a common end point.

$\dagger$ Clearly two such exist, otherwise $M$ contains a punctiform connected set. See my paper, loc. cit. 
In the upper half plane let us construct equilateral triangles with bases $x_{2} y_{2}, y_{4 n-1} y_{4 n+2}, x_{4 n+2} x_{4 n-1},(n=1,2,3, \cdots)$, and in the lower half plane construct equilateral triangles with bases $y_{4 n-3} y_{4 n}$ and $x_{4 n} x_{4 n-3},(n=1,2,3, \cdots)$. Let $T_{0}$ be the set of triangles so constructed.

Now the points $\left(x_{n}\right)$ and $\left(y_{n}\right)$ together with the points of $P_{1}$ divide $I$ into a collection of intervals; let us omit $I_{1}$ from this collection and call $J_{1}$ the resulting collection. Now on each interval of $J_{1}$ let us construct a set of equilateral triangles exactly as we constructed $T_{0}$ on $I$. Let $T_{1}$ be the set of triangles obtained for all intervals of $J_{1}$. Now the vertices of the triangles of $T_{1}$ that are on $I$ together with the points of $P_{2}$ divide $I$ into a set of intervals. Omit from this set the intervals $I_{1}, I_{2}$, $I_{3}$ and call $J_{2}$ the resulting collection. On each interval of $J_{2}$ construct a set of triangles as before and call $T_{2}$ the total set so constructed.

Continue this process indefinitely and let $C=I+\sum_{0}^{\infty} T_{n}$. Then clearly $C$ is a continuum. The maximal free $\operatorname{arcs}$ in $C$ are exactly the intervals $I_{n}$ together with the two sides of each of the triangles of $\left[T_{n}\right]$ not on $I$, so that no two maximal free arcs in $C$ abut. Furthermore the set $R$ of ramification points of $C$ is a subset of $K$ such that $\bar{R}=K$. Thus $\bar{R}$ is totally disconnected and $C$ is a regular curve. Finally we note that if $x$ is any point of $K-(P+R)$, then for each $n$ there is a triangle $t$ of $T_{n}$ with base $a x b$, where $0<a<x<b<1$; and since $\delta(t)<1 /(n+1)$ and $x$ is not a ramification point, it follows that $x$ is not a local separating point. Thus $L \cdot \bar{R}=L \cdot K \subset P+L \cdot R$, which shows that $L \cdot \bar{R}$ is countable, since both $P$ and $L \cdot R$ are countable.

The Johns Hopkins University 